\title{
Perceived force in fatiguing isometric contractions
}

\author{
LYNETTE A. JONES and IAN W. HUNTER \\ McGill University, Montreal, Canada
}

\begin{abstract}
An integrative approach emphasizing both psychological and physiological components in force perception has started to emerge in motor psychophysics. In this experiment, the relation between isometric force (produced by the elbow flexors) and perceived force was examined over a range of forces maintained until maximal endurance. A contralateral-limb matching procedure in which subjects estimated the force of a sustained, constant force contraction by contracting their unfatigued arm at regular intervals was employed. A linear increase in perceived force was observed during the fatiguing contractions, the rate of which depended on the level of force exerted. The sensation of force at maximal endurance was also found to vary with the force exerted. Based on the similarity between these results and those derived from electromyographic studies, we propose that observers use the efferent input to the muscle in preference to its afferent responses in judging the force of muscular contractions.
\end{abstract}

The relation between the perceived force and the physical force exerted in a muscular contraction has frequently been reported to be a power function (e.g., Eisler, 1965; J. C. Stevens \& Mack, 1959). The sensitivity of the perceived-force change to a change in actual force is characterized by the power function's exponent. Typically, experiments purporting to have found a power relation have employed the ratio scaling methods of magnitude estimation and production, in which observers are required to use numerical scales to estimate the magnitude of the force exerted. It has been repeatedly found that the apparent magnitude of force grows as the 1.7 power of the force exerted (Eisler, 1962; J. C. Stevens \& Cain, 1970).

A recent methodological innovation in forceperception research has been the use of contralaterallimb matching procedures (McCloskey, Ebeling, \& Goodwin, 1974). In this paradigm, a muscle group in one limb, termed the reference limb, is perturbed (e.g., changed in length, fatigued, electrically stimulated) and the subject is asked to make the corresponding muscle group in the other limb, called the indicator limb, perceptually the same as some variable (e.g., the force or tension exerted, or the weight supported). This is achieved either by manipulating the variable directly, for example by contracting the muscles in both arms until there is an equal force

Part of this paper was presented at the Society for Neuroscience Annual Meeting in Los Angeles, October 1981. Requests for reprints should be sent to Lynette A. Jones, Department of Psychology, McGill University, 1205 Docteur Penfield Avenue, Montreal, Quebec H3A 1B1, Canada. Ian W. Hunter's mailing address is: Biomedical Engineering Unit, McGill University, 3655 Drummond Street, Montreal, Quebec H3G 1Y6, Canada. sensation, or by informing the experimenter of the changes required to make the limbs the same (e.g., when matching the perceived heaviness of weights). The matching forces or weights chosen by the subject give an indication of the perceived force or heaviness on the reference side. Provided that there is a control matching condition in which an estimate of the observer's baseline accuracy is obtained, it is possible to use this procedure to investigate the perceptual effect of a range of stimuli on the motor system. Some of the problems associated with subjects' linear and nonlinear use of numbers in estimating stimulus magnitude (Poulton, 1981) are avoided with this technique.

When observers match the perceived forces of isometric contractions exerted by the reference and indicator arms, the forces produced by the two limbs are approximately equal under nonfatigued conditions (Cafarelli \& Bigland-Ritchie, 1979; Jones \& Hunter, 1982a). When the length of one muscle is changed and the capacity of that muscle to develop tension is appreciably altered, the subsequent matching function is approximately proportional to the ratio of the indicator to reference muscle maximum voluntary contraction (MVC) (Cafarelli \& BiglandRitchie, 1979). However, if a muscle supporting a weight is partially paralyzed by the regional use of a neuromuscular blocking agent or the antagonist muscle tendon is vibrated during agonist contraction, there is an increase in the perceived heaviness of the weight, as indicated by the matching weights selected by the subject (McCloskey \& Gandevia, 1978). On the basis of these findings from experiments in which the normal relation between cortical and muscular activity has been altered, it has been suggested that it is the centrally generated motor (efferent) command 
sent to the muscle, rather than the afferent signals from muscular, cutaneous, and joint receptors, that is used in estimating the forces exerted by a muscle (McCloskey, 1980).

When a weight is matched simultaneously with a force exerted on a dynamometer, the results differ from those obtained when the observer is matching the force exerted by both arms. J. C. Stevens and Mack (1959) named the former condition crossmodal matching and reported that there was a power function relation between weight and the force of handgrip. The exponent of this function was equal to the ratio of the exponents of the weight to heaviness and muscle-force to perceived-force functions. However, a linear relation was found when the force of foot pressure was matched to the force of handgrip (Eisler, 1962), which indicates that the exponents for these two scales are the same. Matching heaviness with subjective force is not an instance of crossmodal matching, since the sensory systems involved in force and weight perception are essentially the same. The difference in exponent values presumably results from the isotonic nature of lifting weights as compared with the isometric contractions involved in handgrip and foot-pressure exertion. In the former situation, subjects can use the sensory information provided by changes in muscle length in estimating the heaviness of weights.

Changes in perceived force or heaviness occur as a function of the duration of a muscular contraction. It is a frequently experienced phenomenon that the longer one bears a load, the heavier it feels. The perceptual changes that occur when a constant force is maintained over time have been investigated by having observers numerically estimate the magnitude of perceived force after maintaining a contraction for varying periods of time (J. C. Stevens \& Cain, 1970). For static handgrip contractions, it has been proposed that the degree of perceived force (p) grows as a power function of the force of an isometric contraction (f) and also as a power function of the duration $(t)$ of the contraction:

$$
\mathrm{p}=\mathbf{k f}^{1.7} \mathrm{t}^{.7}
$$

where $\mathbf{k}$ is a constant (J. C. Stevens \& Cain, 1970). This equation indicates that for any constant period of time the same exponent (1.7) describes the growth in the perceived force of the contraction, and that the growth of perceived force with duration does not depend upon the force of contraction. Using a magnitude estimation paradigm, Cafarelli, Cain, and J. C. Stevens (1977) found support for this model as applied to the dynamic tasks of cycling and cranking. They noted (as implied by Equation 1) that effort increased much more rapidly with load than with time. Similar increases in perceived force over time were re- ported by McCloskey et al. (1974), who had subjects match with an unfatigued arm the heaviness of a weight supported continuously by the other arm for $15 \mathrm{~min}$. Over this period, the matching weights chosen by the subject gradually increased, so that by the end of the trial the reference weight was overestimated by as much as $70 \%$. Unfortunately, no quantitative analyses of these data were carried out.

The effect of prior fatigue on the perceived force of muscular contractions has also been investigated. Cain and J. C. Stevens (1971) found that fatigue caused a rather uniform increase in the perceived magnitude of force, but exerted little influence on the size of their power function exponent. This suggests that fatigue does not have a differential effect on the observer's sensitivity to force; instead, its effect appears to be uniform across the range of perceptible forces. However, using a cross-modal matching task, Teghtsoonian, Teghtsoonian, and Karlsson (1977) observed that there was a substantial change in the exponent of the power function with fatigue. The effect of fatigue was to make larger forces feel more effortful and smaller forces seem less effortful. They suggested that this latter result may be due to a fatigue-induced elevation in forcedetection threshold, similar to that which occurs with auditory fatigue (Riach, Elliot, \& Reed, 1962).

It has been suggested by several authors, although not experimentally verified, that for any isometric contraction, the sensation of force at the point of maximal endurance is independent of the level of force being maintained (Cafarelli et al., 1977; J. C. Stevens \& Cain, 1970). The present experiment was designed to investigate how perceived force changed as a function of the duration of isometric contractions, and how force was perceived at the point of maximal endurance over a range of forces. Also, the relation between the physiological factors involved in muscle fatigue and the change in perceived force was of interest. A contralateral-limb matching procedure was employed to assess the changes in perceived force over the course of fatiguing isometric contractions.

\section{Method}

\section{Subjects}

Five male and five female university students and staff, from 22 to 31 years of age (mean $=28$ years), participated in this experiment. All gave their informed consent to the procedures described below. All subjects were right-handed and had no known neuromuscular abnormalities of the upper extremities. The mean mass for females and males was $52.1 \mathrm{~kg}( \pm 4.0 \mathrm{~kg})$ and $70.4 \mathrm{~kg}$ $( \pm 9.2 \mathrm{~kg})$, respectively.

\footnotetext{
Apparatus

The experimental sessions took place in a shielded room. The forces produced at the wrists (largely by the biceps brachii muscles) were measured by two strain-gauge force transducers mounted on a wooden and steel frame. A chain was attached to each force transducer, and the distal end of each chain was fitted with a
} 
leather band $(3.6 \mathrm{~cm}$ wide) that was placed around the subject's wrists over the head of the ulna. The subject sat facing the apparatus with vertical and parallel forearms spaced $30 \mathrm{~cm}$ apart, holding a wooden handgrip in each hand and with each elbow joint resting in a molded Sansplint support. The upper arm was horizontal and the hand was in a semipronated position. The angle between the left and right upper arm and forearm was $90 \mathrm{deg}$.

The output from the strain-gauge force transducers was converted and amplified by a strain-gauge bridge (Tektronix Type 3C66), low-pass filtered at $15 \mathrm{~Hz}$ (Grass dc driver amplifier) and then recorded on an $x$-y plotter (Hewlett-Packard 7004B X-Y). The output from the right-arm force transducer was also displayed on a digital voltmeter (Data Precision 935) affixed to the frame directly in front of the subject. This meter served as a monitor for the subject in maintaining reference forces.

\section{Procedure}

Prior to the experimental trials, the subject made a series of MVCs with each arm consecutively. Isometric strength was assessed as the strongest of two brief voluntary maximal contractions $(<3 \mathrm{sec})$. Three minutes were allowed between contractions. The subject participated in four experimental conditions, which were separated by at least $4 \mathrm{~h}$ and usually were conducted on different days. This period of time was necessary for recovery of the endurance capacity of the biceps brachii muscle (Stull \& Kearney, 1978).

The subject was required to maintain a constant force with the right arm at a specified percentage of his or her MVC $(30 \%, 45 \%$, $60 \%$, or $75 \% \mathrm{MVC}$ ). He or she was instructed to maintain a numeric value [the force in newtons $(N)$ of the right arm] on the digital voltmeter. The order of presentation of the four experimental sessions was random. At the start of each session and at 15sec intervals, a 2-sec tone was presented to the subject through headphones. Each time the tone was heard, the subject produced a force with the left arm that he or she perceived to be the same as the force that was currently being exerted with the right arm. After the forces in the arms were matched to the subject's satisfaction (this was accomplished within $5 \mathrm{sec}$ ), the subject relaxed the left arm, but continued maintaining the designated force with the right arm. When the subject was no longer able to maintain this force, such that it was below the specified value for $5 \mathrm{sec}$, his or her maximal endurance time was considered to have been reached and the experimental session was terminated. This paradigm was selected to avoid fatigue of the left (indicator) arm (Jones \& Hunter, 1982b). Prior to each experimental session, the importance of maintaining a contraction for as long as possible was emphasized to each subject. During practice trials before the first session, the subject practiced maintaining small forces, using the digital voltmeter as a reference.

\section{RESULTS}

Due to the sizable individual differences in isometric strength (i.e., MVC) (range $=167-480 \mathrm{~N}$ ), the data were analyzed in both absolute and relative forms. The arithmetic mean of the individual data was calculated to give the group data. There was no significant difference between the left- and right-arm MVC of the subjects ( $p>.05)$. There was, however, a significant difference between males and females in the maximum strength of their left and right arms $[\mathrm{t}(8)=5.5, \mathrm{t}(8)=9.06, \mathrm{p}<.001$, respectively $]$. The average strength of females was $54 \%$ that of the males.

The isometric endurance function of the 10 subjects is shown in Figure 1. The total time that a contraction could be maintained was found to vary in-

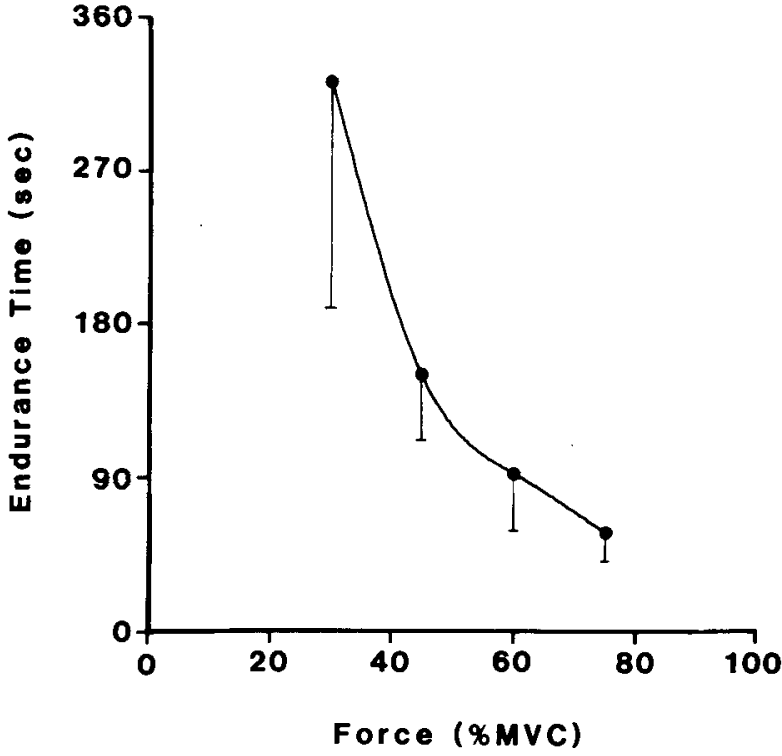

Figure 1. The mean isometric endurance time (with standard deviations) of the elbow flexor muscles of 10 subject at forces raiging from $30 \%$ to $75 \%$ MVC. The cubic spline function fitted to these data is also shown.

versely with the degree of contraction, and there was a correlation of -.75 between maximum strength and the mean endurance time of the four contractions. The greatest variability in endurance times for both males and females occurred at the lowest force level. When the isometric endurance data were plotted on logarithmic coordinates, the slope of the linear function that was fitted by least squares was -1.85 .

Since there were large differences in the durations of contractions for each subject at any one force, the durations were expressed as percentages of the endurance time for each force level. Each subject's matching-force data were fitted by cubic splines (Reinsch, 1971), and 100 equally spaced samples were then taken from each spline function. This method was selected to permit normalization of the results from different subjects and forces, both of which yielded different absolute durations. The last matching trial was taken as the perceived force at $100 \%$ endurance time.

Figure 2 shows the change in the matching forces exerted as the right arm maintained a constant force until maximal endurance. The increase in the matching force during maintained constant force contractions was essentially linear, and the rate of increase depended on the level of constant force exerted, being greater with higher initial forces. However, as Figure 2 illustrates, the increase in matching force within the first minute is considerable even for the smaller forces. There was a significant difference between the matching forces exerted in the four conditions at maximal endurance time $[F(3,27)=6.85$, $\mathrm{p}<.01]$. 
The matching force on the first trial (at zero time) of each experimental session was taken as an estimate of the accuracy of force matching in an unfatigued muscle state. Since there was no significant difference between the left- and right-arm MVC, the matching force exerted by the left arm was expressed as a percentage of that arm's MVC. For the four forces examined $(30 \%, 45 \%, 60 \%$, and $75 \% \mathrm{MVC})$, the perceived forces at zero time were $33 \%, 44 \%$, $58 \%$, and $71 \% \mathrm{MVC}$, respectively. The subjects were able to maintain a constant force accurately during each experimental session. However, their performance became more variable and the task more difficult as the muscle's endurance point approached.

The change in the forces matched to each constant force (expressed as a percentage of each subject's MVC) over the same relative time scale is shown in Figure 3. At maximal endurance time, a force of $30 \%$ MVC was matched with a mean force of $70 \% \mathrm{MVC}$, and a force of $75 \% \mathrm{MVC}$, with a mean force of $90 \%$ MVC. These matching forces exerted at endurance time were significantly different $[\mathrm{F}(3,27)=8.44, \mathrm{p}<$ $.01]$. This result indicates that the perceived force at maximal endurance depends on the level of force exerted. The slopes of the linear functions, fitted by least squares to the force matched as a function of the percentage duration of the contraction, decreased with higher constant forces. The diminishing slope with higher initial forces presumably results from some limitation on the amount the perceived force associated with a force of greater magnitude can increase.

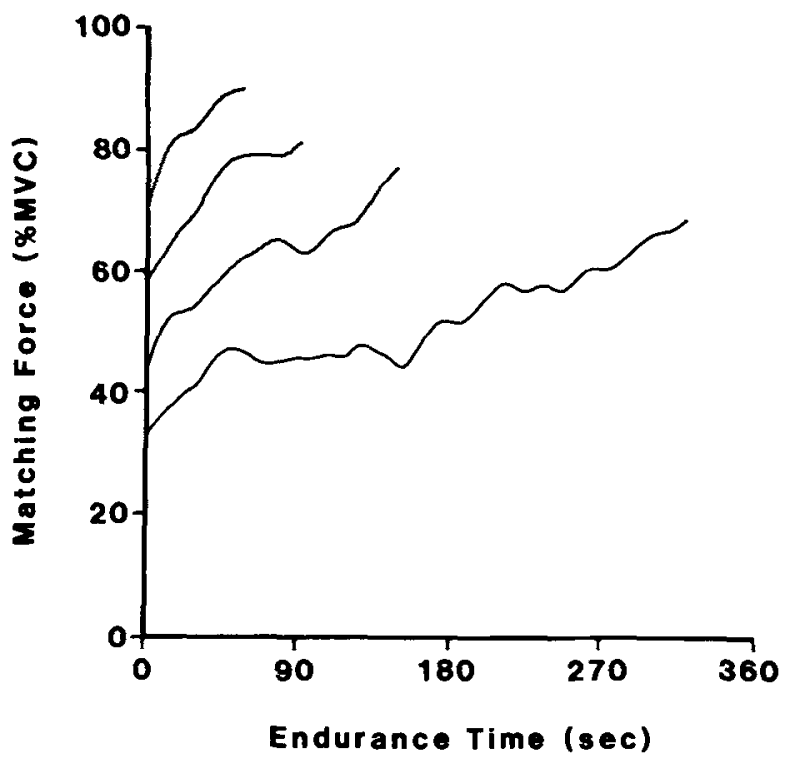

Figure 2. The matching force exerted by the left arm over time for four constant forces $(30 \%, 45 \%, 60 \%$, and $75 \%$ MVC) maintained by the right arm until maximal endurance. The means of the cubic spline functions fitted to the $\mathbf{1 0}$ ubjects' data are shown.

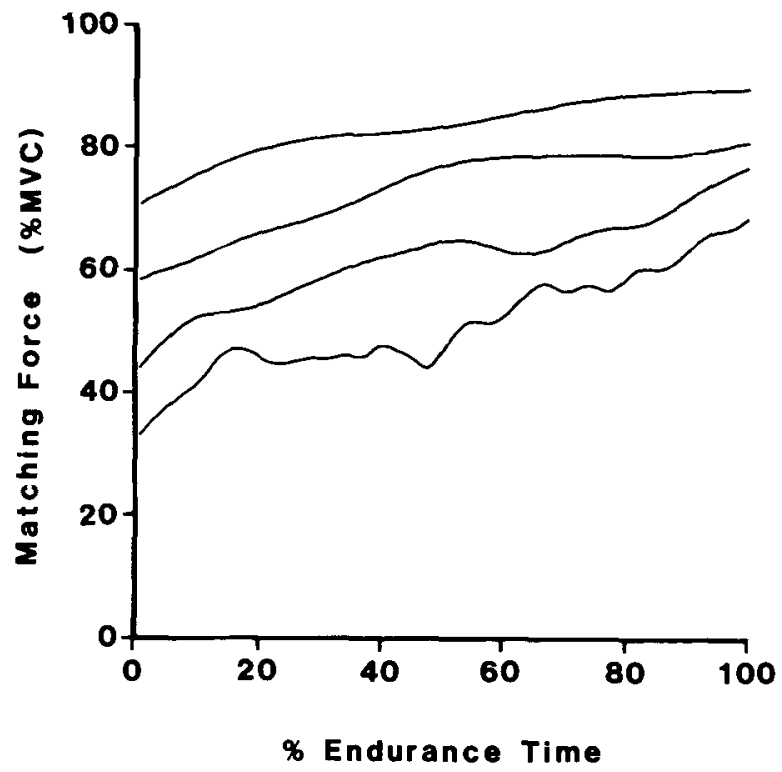

Figure 3. Matching forces exerted by the left arm as a function of the percentage duration of constant force contractions main. tained at $30 \%, 45 \%, 60 \%$, and $75 \%$ MVC. The means of the cubic spline functions fitted to the $\mathbf{1 0}$ subjects' duta are shown.

\section{DISCUSSION}

In previous studies, it has been shown that there is an inverse relation between the force of muscular contraction and isometric endurance (DeVries, 1968; Petrofsky \& Phillips, 1981) and between strength and endurance (Petrofsky, Burse, \& Lind, 1975). Our results confirm these findings. The slope $(-1.85)$ of the endurance function (plotted logarithmically) approximates those reported $(-1.91,-1.85)$ in a number of experiments using different muscle groups (Humphreys \& Lind, 1963; Petrofsky, 1980). The meaning of this relatively invariant feature of the isometric endurance function is uncertain, since the varying biochemical and contractile properties of muscles result in differences in fatigability (Clamann \& Broecker, 1979). It is possible that the fiber composition of the muscle groups investigated has not varied extensively enough for this difference to emerge as a factor in isometric endurance. Most of the isometric endurance functions reported have been obtained from the biceps brachii and finger flexor muscles.

When muscles are unfatigued, the perceived force of an isometric contraction, as measured by a matching contraction, is approximately equal to the force exerted by the contralateral limb. This result confirms those previously reported over this range of forces (Cafarelli \& Bigland-Ritchie, 1979; Jones \& Hunter, 1982a). We did observe a slight tendency towards undermatching forces as the force increased, 
which may have been due to a small but insignificant difference in strength between the arms or to a regression effect (S. S. Stevens \& Greenbaum, 1966). The increase in perceived force that accompanies a constant isometric contraction occurs at a steady rate, with no marked deviations from linearity appearing as the muscle approaches its endurance point. These results differ from those obtained using ratio scaling procedures, with which it has been found that the degree of perceived force grows as a power function of both the isometric force and duration of the contraction (J. C. Stevens \& Cain, 1970). Methodological differences between these two experiments, in particular, the use of the contralaterallimb matching procedure to assess perceived force, presumably account for the different results. In the absence of numerical estimation that requires verbal scaling of magnitude, a linear relation between matching force, isometric force and time emerges.

The changes observed in perceived force are similar to the increases seen in the electrical activity (EMG) of a muscle producing a constant isometric contraction (Petrofsky, 1980; Vredenbregt \& Rau, 1973). The surface EMG is a measure of the excitatory input to a muscle and is an indirect estimate of the magnitude of the descending motor command (Cafarelli \& Bigland-Ritchie, 1979). Petrofsky (1980) observed a linear increase in EMG with sustained contractions and noted that the rate of increase over time depended on the level of force exerted. For forces between $20 \%$ and $70 \% \mathrm{MVC}$, the relative increase in EMG was constant across the same relative time scale, but at forces greater than $70 \% \mathrm{MVC}$, the magnitude of the increase in EMG was progressively less. In the present experiment, the relative increase in perceived force decreased with higher constant forces. However, the marked similarity between the increase in perceived force in this experiment and in EMG under similar conditions provides some support for a centrally mediated theory of force perception. This theory proposes that perception follows the excitatory input to the muscle and is relatively independent of the force exerted. The rapidity with which an increase in perceived force occurs during maintained contractions at higher forces provides additional support for the theory. The biceps brachii muscle has a relatively high percentage of Type II fibers (fast twitch, fast fatiguing), which have little power of endurance (Johnson, Polgar, Weightman, \& Appleton, 1973). At high force levels, motor units composed of these fibers would be brought into activity early during the contraction, and therefore the EMG would increase rapidly.

There are alternative physiological responses that observers could be using in their judgments of the force exerted. Heart rate and mean blood pressure increase during constant isometric contractions, but they both increase nonlinearly over the duration of a contraction and the mean blood pressure increase is independent of the force exerted (Funderburk, Hipskind, Welton, \& Lind, 1974). Muscle spindle afferents are also more active when the efferent input to a muscle increases (Vallbo, 1971), but they do not appear to be responsible for the change in perceived force, since, during muscle tendon vibration, their discharge rate increases and yet the perceived force of contraction is diminished (McCloskey et al., 1974). Joint and cutaneous receptors have been shown to contribute to the perception of force (Gandevia \& McCloskey, 1977), but following joint and skin anesthesia, Roland and Ladegaard-Pedersen (1977) found that judgments of force did not differ from those obtained under normal conditions. Although the present experiment did not address the issue of the relative importance of peripheral sensory receptors in perceived force, it is of interest to establish the physiological basis of force perception. The rate of increase in perceived force during sustained isometric contractions parallels the increase in EMG reported under similar conditions more closely than it does the changes reported in the discharge patterns of intramuscular receptors.

Contrary to the predictions of Cafarelli et al. (1977) and J. C. Stevens and Cain (1970), the sensation of force at maximal endurance depends on the level of force exerted. This suggests that, physiologically, the muscle state varies at the endurance limit and that maximal muscle endurance time is not determined by a perceptual state that is independent of force. The matching forces exerted at maximal endurance ranged from $70 \%$ to $90 \%$ MVC, which is significantly less than the maximum forces the subjects were capable of exerting. This means that the MVC exerted by a fatigued limb is perceptually different from that exerted by an unfatigued one. The EMG at maximal endurance time decreases with smaller constant forces (DeVries, 1968; Petrofsky, 1980), in a manner similar to the perceived force. Evidence that the biochemical state of a muscle at maximal endurance time varies with the level of force comes from the recovery-of-endurance literature. It has been shown that there is a variable rate of recovery in the ability of muscles to sustain exertions following constant force contractions maintained until exhaustion. The recovery is fastest at higher forces and slowest at lower forces (Funderburk et al., 1974; Petrofsky \& Phillips, 1981), and is assumed to depend on the rate of removal of waste products, some of which accumulate in higher concentrations during low force ( $30 \% \mathrm{MVC}$ ) contractions (Karlsson, Funderburk, Essen, \& Lind, 1975).

Using a contralateral-limb matching paradigm, we have shown that the perception of force during constant isometric contractions increases approximately linearly until the muscle reaches its endurance limit, at a rate that depends on the magnitude of 
force exerted. The perceived force at maximal endurance decreases with smaller constant forces, which indicates that the perceived muscle state at maximal endurance changes as a function of contraction duration. The precise relation between the physiological and psychological mechanisms involved in the perception of force remains unclear.

\section{REFERENCES}

Cafarelli, E., \& Bigland-Ritchie, B. Sensation of static force in muscles of different length. Experimental Neurology, $1979,65,511-525$.

Cafarelli, E. W., Cain, W. S., \& Stevens, J. C. Effort of dynamic exercise: Influence of load, duration, and task. Ergonomics, 1977, 20, 147-158.

Cain, W. S., \& Stevens, J. C. Effort in sustained and phasic handgrip contractions. American Journal of Psychology, 1971, 84, 52-65.

Clamann, H. P., \& Broecker, K. T. Relation between force and fatigability of red and pale skeletal muscles in man. American Journal of Physical Medicine, 1979, 58, 70-85.

DeVnies, H. A. Method for evaluation of muscle fatigue and endurance from electromyographic fatigue curves. American Journal of Physical Medicine, 1968, 47, 125-135.

Eisler, H. Subjective scale of force for a large muscle group. Journal of Experimental Psychology, 1962, 64, 253-257.

Eislen, H. The ceiling of psychophysical power functions. American Journal of Psychology, 1965, 78, 506-509.

Funderburk, C. F., Hipsinind, S. G., Welton, R. C., \& Lind, A. R. Development of and recovery from fatigue induced by static effort at various tensions. Journal of Applied Physiology, $1974,37,392-396$.

Gandevia, S. C., \& McCloskey, D. I. Effects of related sensory inputs on motor performances in man studied through changes in perceived heaviness. Journal of Physiology, 1977, 272, 653-672.

HumphreYs, P. W., \& Lind, A. R. The blood flow through active and inactive muscles of the forearm during sustained hand-grip contractions. Journal of Physiology, 1963, 166, 120-135.

Johnson, M. A., Polgar, J., Weightman, D., \& Appleton, D. Data on the distribution of fiber types in thirty-six human muscles. An autopsy study. Journal of the Neurological Sciences, 1973, 18, 111-129.

Jones, L. A., \& Hunter, I. W. Force sensation in isometric contractions: A relative force effect. Brain Research, 1982, 244, 186-189. (a)

Jones, L. A., \& Hunten, I. W. The relation of muscle force and EMG to perceived force in human finger flexors. European Journal of Applied Physiology, 1982, 50, 125-131. (b)

Karlsson, J., Funderburk, C. F., Essen, B., \& Lind, A. R. Constituents of human muscle in isometric fatigue. Journal of Applied Physiology, 1975, 38, 208-211.

McCloskeY, D. I. Kinaesthetic sensations and motor commands in man. In J. E. Desmedt (Ed.), Spinal and supraspinal mechanisms of voluntary motor control and locomotion (Vol. 8). Basel: Karger, 1980.

McCloskey, D. I., Ebeling, P., \& Goodwin, G. M. Estimation of weights and tensions and apparent involvement of a "sense of effort." Experimental Neurology, 1974, 42, 220-232.

McCloskey, D. I., \& Gandevia, S. C. Role of inputs from skin, joints and muscles and of corollary discharges, in human discriminatory tasks. In G. Gordon (Ed.), Active touch: The mechanism of recognition of objects by manipulation. Oxford: Pergamon Press, 1978.

Petrofsky, J. S. Computer analysis of the surface EMG during isometric exercise. Computers in Biology and Medicine, 1980, 10, 83-95.

Petrofsky, J. S., Burse, R. L., \& Lind, A. R. Comparison of physiological responses of women and men to isometric exercise. Journal of Applied Physiology, 1975, 38, 863-868.

Petrofsky, J. S., \& Phillips, C. A. The influence of body fat on isometric exercise performance. Ergonomics, 1981, 24, 215-222.

Poulton, E. C. Schooling and the new psychophysics. Behavioral and Brain Sciences, 1981, 4, 201-203.

REINsch, C. H. Smoothing by spline functions. II. Numerische Mathematik, 1971, 16, 451-454.

Riach, W., Elliot, D. N., \& REed, J. C. Growth of loudness and its relationship to intensity discrimination under various levels of auditory fatigue. Journal of the Acoustical Society of America, 1962, 34, 1764-1767.

Roland, P. E., \& Ladegandd-Pedersen, H. A quantitative analysis of sensations of tensions and of kinaesthesia in man. Brain, 1977, 100, 671-692.

Stevens, J. C., \& Cain, W. S. Effort in isometric muscular contractions related to force level and duration. Perception \& Psychophysics, 1970, 8, 240-244.

Stevens, J. C., \& MACK, J. D. Scales of apparent force. Journal of Experimental Psychology, 1959, 58, 405-413.

Stevens, S. S., \& Greenbaum, H. B. Regression effect in psychophysical judgment. Perception \& Psychophysics, 1966, $1,439-446$.

StULL, G., \& KEARNEY, J. Recovery of muscular endurance following submaximal isometric exercise. Medicine and Science in Sports, 1978, 10, 109-112.

Teghtsoonian, R., Teghtgoonian, M., \& Karlsson, J. G. The effects of fatigue on the perception of muscular effort. In G. Borg (Ed.), Physical work and effort. Oxford: Pergamon Press, 1977.

VALLBO, A. B. Muscle spindle response at the onset of isometric voluntary contractions in man. Time difference between fusimotor and skeletomotor effects. Journal of Physiology, 1971, $318,405-431$.

VRedenbreat, J., \& RAU, G. Surface electromyography in relation to force, muscle length and endurance. In J. E. Desmedt (Ed.), New developments in electromyography and clinical neurophysiology (Vol. 1). Basel: Karger, 1973.

(Manuscript received October 8, 1981; revision accepted for publication December 16,1982 .) 